\title{
Real-Time Surgical Simulation Using Reduced Order Finite Element Analysis
}

\author{
Zeike A. Taylor ${ }^{1,2}$, Stuart Crozier ${ }^{1}$, and Sébastien Ourselin ${ }^{2}$ \\ 1 MedTeQ Centre, School of Information Technology \& Electrical Engineering, \\ The University of Queensland, Brisbane, QLD, 4072, Australia \\ 2 Centre for Medical Image Computing, University College London, \\ Gower St, London, WC1E 6BT, UK \\ ztaylor@itee.uq.edu.au
}

\begin{abstract}
Reduced order modelling, in which a full system response is projected onto a subspace of lower dimensionality, has been used previously to accelerate finite element solution schemes by reducing the size of the involved linear systems. In the present work we take advantage of a secondary effect of such reduction for explicit analyses, namely that the stable integration time step is increased far beyond that of the full system. This phenomenon alleviates one of the principal drawbacks of explicit methods, compared with implicit schemes. We present an explicit finite element scheme in which time integration is performed in a reduced basis. The computational benefits of the procedure within a GPU-based execution framework are examined, and an assessment of the errors introduced is given. Speedups approaching an order of magnitude are feasible, without introduction of prohibitive errors, and without hardware modifications. The procedure may have applications in medical image-guidance problems in which both speed and accuracy are vital.
\end{abstract}

\section{Introduction}

In the last decade significant effort has been devoted to use of computational biomechanics for enhancing the utility of medical images in the operating theatre. A typical scenario is the use of information-rich pre-operative images to guide interventions. Such images may, for example, delineate target pathologies or vasculature and other vital structures. However, if the relevant biological structures change shape between imaging and intervention, guidance may be confounded unless the deformation can be reliably compensated for. Since soft tissues are, of course, physical structures, their deformation must conform to physical laws, and this has motivated use of biomechanics to this end [1].

A common issue is the stringent time constraint on simulations performed intra-operatively, which competes with requirements for accuracy and reliability in the solution. Continuum mechanics formalism, combined with finite element solution, is the most common framework. However, despite the maturity of nonlinear formulations in the biomechanics community, these have seldom been used, largely due to the mentioned time constraints.

T. Jiang et al. (Eds.): MICCAI 2010, Part II, LNCS 6362, pp. 388-395, 2010.

(C) Springer-Verlag Berlin Heidelberg 2010 
Recently, explicit dynamic algorithms have been shown to be advantageous in this area [1213], but a well-known drawback of such methods is the small time steps required for numerical stability. By virtue of its dependence on material stiffness, the critical step size $\Delta t_{\text {cr }}$ is much larger for models of very soft tissues (such as organs) than of common engineering materials, but is still many times smaller than that required in implicit analyses. In the present work, we examine the use of reduced order models (ROMs) to alleviate some of the computational load in explicit analyses, and thereby improve their utility.

The main idea behind ROMs is projection of the full model response onto a lower dimensional reduced basis. In a displacement-based finite element context, the complete displacement field is approximated by a set of generalised variables of much lower dimension. In most developments in this area 68 , the key benefit is the reduction in the size of the linear system to be solved at each step, since mass and stiffness matrices assume the dimensionality of the reduced system. However, in explicit analyses, these large matrices are removed, and the utility of the ROMs is less obvious. Our work makes use of a secondary effect for explicit methods: by performing the time integration in the reduced basis, a much larger solution time step may be used [6]. This phenomenon does not appear to have been exploited since its discovery by Krysl and co-workers, despite its potential benefits.

In the following, we describe an explicit solution method which incorporates integration in the reduced basis, as described. The computational benefits of the procedure within a GPU-based execution framework are examined, subsequently, along with the errors incurred.

\section{A Reduced Order Explicit Dynamic FE Algorithm}

In the following we draw on the work of Taylor et al. 12 13, in which an explicit dynamic FE algorithm was employed. We refer the reader to these publications for further algorithm details.

\subsection{Equilibrium Equations}

We are concerned with solution of the standard equilibrium equations for a nonlinear, dynamic, damped finite element model:

$$
\mathbf{M} \ddot{\mathbf{U}}+\alpha \mathbf{M} \dot{\mathbf{U}}+\mathbf{K}(\mathbf{U}) \mathbf{U}=\mathbf{F}^{\mathrm{ext}},
$$

where $\mathbf{M}$ is the (diagonalised) mass matrix, $\alpha$ is a damping coefficient, $\mathbf{K}(\mathbf{U})$ is the stiffness matrix, $\mathbf{F}^{\text {ext }}$ is a vector of external loads, and $\mathbf{U}$ is a vector of nodal displacements. When explicit time integration is used, the stiffness term $\mathbf{K}(\mathbf{U}) \mathbf{U}$ may be evaluated element-wise and converted into an equivalent vector of internal forces $\mathbf{F}^{\text {int }}$ (see 12 for details). Thus, at time increment $n$, the equilibrium equations read

$$
\mathbf{M} \ddot{\mathbf{U}}_{n}+\alpha \mathbf{M} \dot{\mathbf{U}}_{n}=\mathbf{F}_{n}^{\mathrm{eff}},
$$

where $\mathbf{F}_{n}^{\text {eff }}=\mathbf{F}_{n}^{\text {ext }}-\mathbf{F}_{n}^{\text {int }}$ is called the effective load vector. 


\subsection{Generalised Displacements and the Reduced Basis}

The system defined by Eqn. (2), referred to as the full system, has dimension $N$, and its response is encapsulated by the nodal displacements $\mathbf{U}$. For the present case of a displacement-based finite element method in 3D, we have $N=3 N_{\text {nodes }}$, where $N_{\text {nodes }}$ is the number of nodes. The key idea of reduced order modelling in a finite element context is to approximate the full response with a set of generalised displacements $\mathbf{P}$ of much lower dimension $M$ :

$$
\mathbf{U}=\mathbf{\Phi P},
$$

where $\boldsymbol{\Phi}$ is referred to as the reduced basis. $\boldsymbol{\Phi}$ is time-independent, thus: $\dot{\mathbf{U}}=\mathbf{\Phi} \dot{\mathbf{P}}$, $\ddot{\mathbf{U}}=\boldsymbol{\Phi} \ddot{\mathbf{P}}$. Substituting these into (2) and projecting on $\boldsymbol{\Phi}$ yields

$$
\hat{\mathbf{M}} \ddot{\mathbf{P}}_{n}+\alpha \hat{\mathbf{M}} \dot{\mathbf{P}}_{n}=\hat{\mathbf{F}}_{n}^{\mathrm{eff}},
$$

in which we have introduced a reduced mass matrix $\hat{\mathbf{M}}=\boldsymbol{\Phi}^{\mathrm{T}} \mathbf{M} \boldsymbol{\Phi}$ and reduced effective load $\hat{\mathbf{F}}^{\text {eff }}=\boldsymbol{\Phi}^{\mathrm{T}} \mathbf{F}^{\mathrm{eff}}$. Eqn. (4) constitutes an equivalent equilibrium equation for the reduced system.

\subsection{Computing the Reduced Basis}

For a full-order dynamic FE model subjected to some loading, we define a matrix $\overline{\mathbf{U}}$ of snapshots:

$$
\overline{\mathbf{U}}=\left[\mathbf{u}_{1}-\overline{\mathbf{u}} \mathbf{u}_{2}-\overline{\mathbf{u}} \ldots \mathbf{u}_{S}-\overline{\mathbf{u}}\right]
$$

where $\mathbf{u}_{i}(i=1, \ldots, S)$ are $N$-vectors of nodal displacements captured at $S$ different time points, and $\overline{\mathbf{u}}=\left(\sum_{i=1}^{S} \mathbf{u}_{i}\right) / S$ is their mean. $\mathbf{u}_{i}$ are referred to as snapshots, and we call the collection of them the training set for the reduced model. We seek an optimal $M$-dimensional approximation for the response history $\overline{\mathbf{U}}$, with $M \ll N$. It may be shown that this is given by the eigenvectors $\left.\phi_{i}(i=1, \ldots, M)\right)^{1}$ corresponding to the $M$ largest eigenvalues of the covariance matrix $\mathbf{C}_{d}=\overline{\mathbf{U}} \overline{\mathbf{U}}^{\mathrm{T}} / M[6] 8$. The desired reduced basis $\boldsymbol{\Phi}$ is then given by

$$
\boldsymbol{\Phi}=\left[\phi_{1} \phi_{2} \ldots \phi_{M}\right] .
$$

The covariance matrix $\mathbf{C}_{d}$ is $N \times N$. Hence, for practical problems, obtaining the required eigenvectors is computationally intensive. For $S<N$ (which is usual) a more efficient approach exists. Begin by computing the first $M$ eigenvectors $\boldsymbol{\psi}_{i}$ of the smaller matrix $\mathbf{C}_{s}=\overline{\mathbf{U}}^{\mathrm{T}} \overline{\mathbf{U}} / M$. The corresponding eigenvalues are denoted $\lambda_{i}$. Scale each eigenvector according to $\overline{\boldsymbol{\psi}}_{i}=\boldsymbol{\psi}_{i} / \sqrt{\lambda_{i}}$. The desired basis is then recovered by projecting back on the matrix of snapshots: $\boldsymbol{\Phi}=\overline{\mathbf{U}} \overline{\boldsymbol{\Psi}}$, where $\overline{\boldsymbol{\Psi}}$ is a matrix whose columns are the scaled vectors $\bar{\psi}_{i}$.

In the study in [6], the reduced models were trained using snapshots from a single load case. Models so constructed are able to reproduce very well the full response to this load, but are less accurate when subjected to different loads. We have found that the robustness of the reduced models may be greatly improved by including snapshots from several different load cases in the training set.

\footnotetext{
${ }^{1}$ We refer to these as the first $M$ eigenvectors.
} 


\subsection{Explicit Integration in the Reduced Basis}

In an incremental nonlinear analysis, we require a procedure for computing the new model configuration at each step. Assume, temporarily, that the generalised displacements $\mathbf{P}_{n-1}$ and $\mathbf{P}_{n}$ for the previous and current steps, respectively, are known, and the reduced effective load $\hat{\mathbf{F}}_{n}^{\text {eff }}$ has been computed. We define discrete approximations for current generalised accelerations and velocities, $\ddot{\mathbf{P}}_{n}=\left(\mathbf{P}_{n-1}-2 \mathbf{P}_{n}+\mathbf{P}_{n+1}\right) / \Delta t^{2}$ and $\dot{\mathbf{P}}_{n}=\left(\mathbf{P}_{n+1}-\mathbf{P}_{n-1}\right) / 2 \Delta t$, respectively, in which $\Delta t$ is the solution time step. Substituting these in the equilibrium equation (4) and rearranging yields an expression for the next set of generalised displacements:

$$
\mathbf{P}_{n+1}=\gamma_{1} \hat{\mathbf{M}}^{-1} \hat{\mathbf{F}}_{n}^{\mathrm{eff}}+\gamma_{2} \mathbf{P}_{n}+\gamma_{3} \mathbf{P}_{n-1},
$$

where $\gamma_{1}=2 \Delta t^{2} /(\alpha \Delta t+2), \gamma_{2}=2 \gamma_{1} / \Delta t^{2}$, and $\gamma_{3}=1-\gamma_{2}$ are constants. Then, the full displacements may be recovered by multiplying through by $\boldsymbol{\Phi}$ :

$$
\begin{aligned}
\mathbf{U}_{n+1} & =\boldsymbol{\Phi} \mathbf{P}_{n+1} \\
& =\gamma_{1} \boldsymbol{\Phi} \hat{\mathbf{M}}^{-1} \hat{\mathbf{F}}_{n}^{\mathrm{eff}}+\gamma_{2} \boldsymbol{\Phi} \mathbf{P}_{n}+\gamma_{3} \boldsymbol{\Phi} \mathbf{P}_{n-1} \\
& =\gamma_{1} \boldsymbol{\Phi} \hat{\mathbf{M}}^{-1} \boldsymbol{\Phi}^{\mathrm{T}} \mathbf{F}_{n}^{\mathrm{eff}}+\gamma_{2} \mathbf{U}_{n}+\gamma_{3} \mathbf{U}_{n-1}
\end{aligned}
$$

$\hat{\mathbf{M}}$ is a dense matrix, but of much smaller size $(M \times M$ where $M \ll N)$ than the full mass matrix M. In practice its inverse is easily precomputed and stored, and occupies minimal storage. A more pertinent issue from the point of view of parallelisation is that the displacement update is no longer a simple vector equation. This is addressed in Sect. 3.

The new displacement update procedure (8) consists of projecting the effective load on the reduced basis, evaluating a generalised acceleration term, and projecting back on the full system. As mentioned, the principal benefit of doing so is that it increases the stable time step far beyond that of the full system. While the precise mechanism for this has not been established, it is likely that by evaluating the accelerations in the reduced basis we remove many of the higher frequency modes of the full system, thus increasing the effective minimum freevibrational period on which the critical limit depends. Many of these frequencies result from discretisation artifacts and do not contribute meaningful information to the solution. Many others, though physical, may be neglected also without significantly altering the solution, as will be seen.

\subsection{Consistency of the Nonlinear Formulation}

It is important to note that the only modification of the algorithm in 1213 . is in the time integration procedure; the evaluation of stresses at integration points and their integration to obtain internal forces $\mathbf{F}^{\text {int }}$ are unchanged. Thus, assuming Eqn. (8) provides a sufficiently accurate approximation for the full displacements, the large deformation consistency of the nonlinear formulation is intact. 


\section{GPU Implementation Using CUDA}

Using the CUDA API 9], the proposed algorithm was implemented for GPU execution. As mentioned, the introduction of the matrix multiplications in the displacement update (8) complicates parallel execution. Instead of the single computation kernel for this process used in [1213, we require several. Execution is divided into precomputation and time-loop phases. The precomputation phase is as described in [12[13] except for the computation of the inverse reduced mass matrix $\hat{\mathbf{M}}^{-1}$. At each step in the time-loop the execution procedure is now:

1. Compute internal forces $\mathbf{F}_{n}^{\text {int }}$ [GPU]

2. Compute effective loads $\mathbf{F}_{n}^{\text {eff }}=\mathbf{F}_{n}^{\text {ext }}-\mathbf{F}_{n}^{\text {int }}[\mathbf{G P U}]$

3. Transfer $\mathbf{F}_{n}^{\text {eff }}$ to host

4. Compute reduced effective loads $\hat{\mathbf{F}}_{n}^{\text {eff }}=\boldsymbol{\Phi}^{\mathrm{T}} \mathbf{F}_{n}^{\text {eff }}[\mathbf{C P U}]$

5. Compute $\hat{\mathbf{F}}_{n}^{\text {eff }}=\hat{\mathbf{M}}^{-1} \hat{\mathbf{F}}_{n}^{\text {eff }}[\mathbf{C P U}]$

6. Compute $\mathbf{U}_{n+1}=\boldsymbol{\Phi} \hat{\mathbf{F}}_{n}^{\text {eff }}[\mathbf{C P U}]$

7. Transfer $\mathbf{U}_{n+1}$ to device

8. Compute $\mathbf{U}_{n+1}=\gamma_{1} \mathbf{U}_{n+1}+\gamma_{2} \mathbf{U}_{n}+\gamma_{3} \mathbf{U}_{n-1}$ [GPU]

Bold, bracketed comments indicate use of CPU or GPU execution. Notably, steps 4-6 are executed on the CPU, which, owing to the small sizes of the involved quantities, we found to be faster than invoking additional CUDA kernel launches. Steps 1, 2, and 8 are implemented as CUDA kernel functions. Thus, the new algorithm requires three kernel launches (plus two data transfers and some additional CPU computations), compared with two for the full model implementation 1213 .

\section{Assessment Using a Brain Shift Model}

We assessed the robustness and computational performance of the presented algorithm using a model of the well-known brain shift problem 4. Many methods for compensating for the brain motion using finite element analysis have been proposed [37/10/14. We studied a synthetic scenario in which the skull is imagined to be opened adjacent to the right frontal lobe, and brain motion is induced in this region.

The brain tissue was modelled as a neo-Hookean hyperelastic material with shear modulus $\mu=1 \mathrm{kPa}$ and bulk modulus $\kappa=50 \mathrm{kPa}$ 14. A model comprising 7480 nodes and 39323 tetrahedral elements (non-locking Averaged Nodal Pressure formulation was used [5]) was employed. Loads were applied over 1sec of simulated time (sufficiently slow to obtain an approximately stable static solution) to a group of nodes on the right frontal lobe, and surface nodes on the opposite side of the model (left, posterior) were assumed to be in contact with the skull and were fixed. In the present context, only the final deformed configuration of the brain is of interest.

We emphasise that this study is not based on a particular clinical case, and is intended solely to enable comparison of the reduced order modelling approach with the well-established regular approach for a clinically relevant scenario. That is, we seek to establish whether the same results are obtained with each method. 


\subsection{A Priori Construction of the Reduced Basis}

Reduced bases $\boldsymbol{\Phi}$ were constructed by compiling full model response snapshots from five independent load cases. The applied loads in each case were of similar magnitude, but varying direction, with the directions covering an angular range of $40^{\circ} .100$ snapshots were extracted from each case, yielding a training set size of 500 . To study the effects of varying basis size, bases with $M=4, \ldots, 10$ were constructed. For the studied scenario it was found that errors became impractically large for $M<4$, and decreased extremely slowly for $M>10$.

\subsection{Performance of the Reduced Order Models}

The full and reduced order models were subjected to three test load cases, randomly generated from the range of the training set loads.

Critical Time Step and Solution Time. The variation in the critical time step $\Delta t_{\text {cr }}$ with basis size is plotted in Fig. 1. Generally, as the basis size $M$ increases, the time step decreases, approaching the full model value. The concomitant improvement in solution time for each basis is shown also. For the current scenario, a peak speedup of 13.4 times was obtained for $M=4$. Even using the largest basis $(M=10)$ resulted in a speedup of 4.3 times. The gap between the time step improvement and the corresponding solution time improvement is indicative of the greater computational cost per step of the new algorithm. We estimate the step-wise cost to be between 1.7 (for $M=4$ ) and 2.3 (for $M=10$ ) times greater for the new algorithm. As observed, though, this cost is outweighed by the step size increase nonetheless. The proportion of the overall computation time spent on each algorithm step is shown in Fig. 1, also.
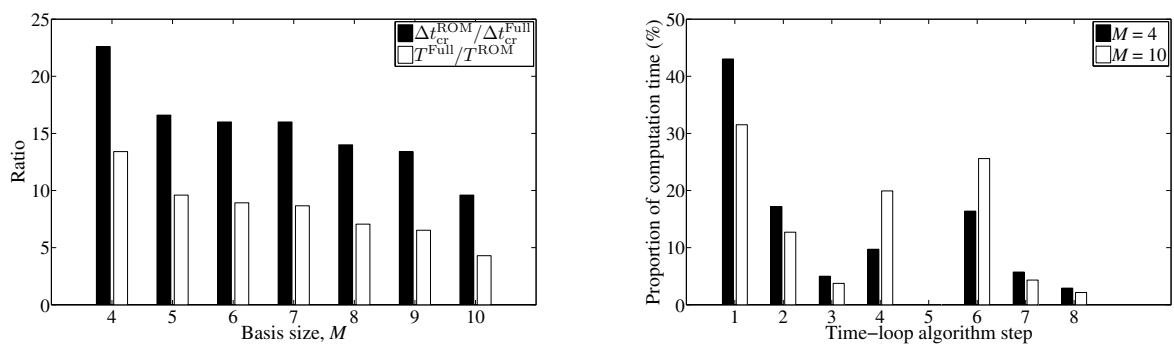

Fig. 1. (a) Left: Ratios of reduced order model critical time step $\Delta t_{\mathrm{cr}}^{\mathrm{ROM}}$ to full model step $\Delta t_{\mathrm{cr}}^{\mathrm{Full}}$, and ratios of full model overall solution time $T^{\text {Full }}$ to reduced model time $T^{\text {ROM }}$, as functions of basis size. Right: Proportion of the computation time taken for each time-loop algorithm step (Sect. 3) for the smallest and largest bases.

Solution Errors. The nodal solution errors for each load case and each basis size are shown in Fig. 2. For $M<7$, errors began to rise more sharply, though the 
maximum value over all simulations was still $<0.6 \mathrm{~mm}$. Using $M \geq 5$, ensured relatively low errors in all cases, while still affording a solution speedup of 9.6 times. Given typical brain MR image resolutions are around $1 \mathrm{~mm}$, we feel these error levels are acceptable for medical imaging applications, and are, moreover, within the realistically obtainable accuracy of full models 3/7,1011/14. The distribution of error over the mesh for load case 3 is shown in Fig. (2), also.
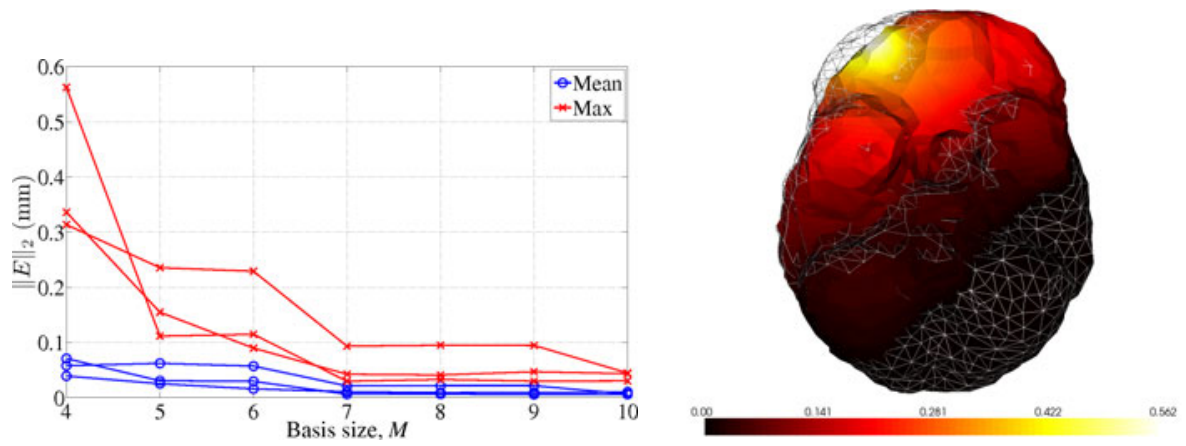

Fig. 2. Left: Mean and maximum nodal error magnitudes as a function of basis size for the three test load cases. Right: Inferior view of the deformed mesh for load case 3 (worst case) using the smallest basis $(M=4)$, with colours mapped to error magnitude (units are $\mathrm{mm}$ ). A wireframe model of the undeformed mesh is overlaid.

\section{Discussion and Conclusions}

We have presented a reduced order nonlinear explicit finite element algorithm for soft tissue simulation, and described its implementation for graphics hardware execution. Using a basis size of $M=5$, the method affords solution speedups of nearly an order of magnitude, while confining errors with respect to the full model to less than $0.24 \mathrm{~mm}$ (corresponding to about $1-2 \%$ of the maximum deformation). For the example brain model investigated, overall solution times were approximately $500 \mathrm{~ms}$ for a simulated time of $1 \mathrm{sec}$ - hence, real-time solution was comfortably achieved. While GPU-based execution was employed, it should be noted that these speed improvements result entirely from algorithmic modifications, not hardware differences.

A drawback of the current algorithm is its support for homogeneous essential boundary conditions, only. In this study, loading was applied via natural (force-based) boundary conditions. However, as noted in [6], methods exist for converting essential boundary conditions into equivalent natural ones. Penalty methods, which form the basis of widely used penalty contact formulations, are one such example. Our current work involves development of efficient procedures for this purpose.

Our approach may have applications in motion compensation for imageguided therapies, for which fast and accurate solutions are required. Particularly relevant examples include compensation for brain motion during neurosurgery, 
as described, and for respiratory motion during lung radiotherapy [2]. In both applications, the loading is approximately known prior to the intervention, allowing reasonable training sets to be developed. Solutions for the precise loadings emerging intra-operatively may then be obtained rapidly and with a high level of confidence.

\section{References}

1. Carter, T.J., Sermesant, M., Cash, D.M., Barratt, D.C., Tanner, C., Hawkes, D.J.: Application of soft tissue modelling to image-guided surgery. Medical Engineering \& Physics 27(10), 893-909 (2005)

2. Colgan, R., McClelland, J., McQuaid, D., Evans, P.M., Hawkes, D., Brock, J., Landau, D., Webb, S.: Planning lung radiotherapy using 4D CT data and a motion model. Physics in Medicine and Biology 53, 5815-5830 (2008)

3. Ferrant, M., Nabavi, A., Macq, B., Jolesz, F., Kikinis, R., Warfield, S.: Registration of 3-D intraoperative MR images of the brain using a finite-element biomechanical model. IEEE Transactions on Medical Imaging 20(12), 1384-1397 (2001)

4. Hill, D.L.G., Maurer, C.R., Maciunas, R.J., Barwise, J.A., Fitzpatrick, J.M., Wang, M.Y.: Measurement of intraoperative brain surface deformation under a craniotomy. Neurosurgery 43(3), 514-526 (1998)

5. Joldes, G.R., Wittek, A., Miller, K.: Non-locking tetrahedral finite element for surgical simulation. Communications in Numerical Methods in Engineering 25(7), 827-836 (2009)

6. Krysl, P., Lall, S., Marsden, J.E.: Dimensional model reduction in non-linear finite element dynamics of solids and structures. International Journal for Numerical Methods in Engineering 51, 479-504 (2001)

7. Miga, M.I., Paulsen, K.D., Lemery, J.M., Eisner, S.D., Hartov, A., Kennedy, F.E., Roberts, D.W.: Model-updated image guidance: initial clinical experiences with gravity-induced brain deformation. IEEE Transactions on Medical Imaging 18(10), 866-874 (1999)

8. Niroomandi, S., Alfaro, I., Cueto, E., Chinesta, F.: Real-time deformable models of non-linear tissues by model reduction techniques. Computer Methods and Programs in Biomedicine 91, 223-231 (2008)

9. NVIDIA Corporation: NVIDIA CUDA Programming Guide Version 2.3 (2009)

10. Skrinjar, O., Nabavi, A., Duncan, J.: Model-driven brain shift compensation. Medical Image Analysis 6(4), 361-373 (2002)

11. Tanner, C., Schnabel, J.A., Leach, M.O., Hose, D.R., Hill, D.L.G., Hawkes, D.J.: Factors influencing the accuracy of biomechanical breast models. Medical Physics 33(6), 1758-1769 (2006)

12. Taylor, Z.A., Cheng, M., Ourselin, S.: High-speed nonlinear finite element analysis for surgical simulation using graphics processing units. IEEE Transactions on Medical Imaging 27(5), 650-663 (2008)

13. Taylor, Z.A., Comas, O., Cheng, M., Passenger, J., Hawkes, D.J., Atkinson, D., Ourselin, S.: On modelling of anisotropic viscoelasticity for soft tissue simulation: numerical solution and GPU execution. Medical Image Analysis 13(2), 234-244 (2009)

14. Wittek, A., Miller, K., Kikinis, R., Warfield, S.K.: Patient-specific model of brain deformation: Application to medical image registration. Journal of Biomechanics 40(4), 919-929 (2007) 